Theoretical and Mathematical Physics, 172(2): 1147-1159 (2012)

\title{
ENVELOPE SOLITON RESONANCES AND BROER-KAUP-TYPE NON-MADELUNG FLUIDS
}

(c) O. K. Pashaev*

\begin{abstract}
We derive an extended nonlinear dispersion for envelope soliton equations and also find generalized equations of the nonlinear Schrödinger (NLS) type associated with this dispersion. We show that space dilatations imply hyperbolic rotation of the pair of dual equations, the NLS and resonant NLS (RNLS) equations. For the RNLS equation, in addition to the Madelung fluid representation, we find an alternative non-Madelung fluid system in the form of a Broer-Kaup system. Using the bilinear form for the RNLS equation, we construct the soliton resonances for the Broer-Kaup system and find the corresponding integrals of motion and existence conditions for the soliton resonance and also a geometric interpretation in terms of a pseudo-Riemannian surface of constant curvature. This approach can be extended to construct a resonance version and the corresponding Broer-Kaup-type representation for any envelope soliton equation. As an example, we derive a new modified Broer-Kaup system from the modified NLS equation.
\end{abstract}

Keywords: soliton resonance, Madelung fluid, Broer-Kaup system, envelope soliton, resonant NLS

\section{Introduction}

The Madelung fluid representation of the Schrödinger equation is an important tool, first created for interpreting quantum mechanics [1], [2] and then realized as a quantum fluid model [3] and later as a proper tool for describing the semiclassical limit of envelope soliton equations of the nonlinear Schrödinger (NLS) type [4], [5]. Here, we use it to generalize the NLS equation by extending the dispersion term. This generalization preserves the integrability and admits a special range of parameters with resonance soliton interactions. By dilatation of space coordinates, we show that the NLS equation is related to its dual resonance equation by a hyperbolic rotation. This dual model, the resonant NLS (RNLS) equation [6], in addition to the Madelung form with a quantum potential term, can be rewritten for the drift velocity and becomes the Broer-Kaup system. In contrast to the trilinear form known for the Broer-Kaup model [7], we obtain a bilinear form for describing soliton resonances of the Broer-Kaup system in this approach. In general, our approach allows constructing a resonance version and the corresponding Broer-Kaup-type representation for any envelope soliton equation. As an illustration, we construct a new modified BroerKaup model for the modified NLS (MNLS) equation.

1.1. Complex Burgers equation. In his first short communication in 1926 on the hydrodynamic interpretation of the Schrödinger equation [1], Madelung introduced the complex velocity field

$$
\mathbf{V}=\frac{\hbar}{m} \frac{\nabla \Psi}{\Psi}=\mathbf{v}_{\mathrm{q}}+i \mathbf{v}_{\mathrm{c}}
$$

where the imaginary part is interpreted as the electron velocity, which coincides with the Madelung-LandauLondon definition of the local mean velocity [8]

*Department of Mathematics, Izmir Institute of Technology, Izmir, Turkey, e-mail: oktaypashaev@iyte.edu.tr.

Prepared from an English manuscript submitted by the author; for the Russian version, see Teoreticheskaya $i$ Matematicheskaya Fizika, Vol. 172, No. 2, pp. 308-322, August, 2012. 


$$
\mathbf{v}_{\mathrm{c}}=\frac{i \hbar}{2 m \rho}(\Psi \nabla \bar{\Psi}-\bar{\Psi} \nabla \Psi)=\frac{\hbar}{m} \Im \frac{\nabla \Psi}{\Psi}=\frac{\nabla S}{m},
$$

where $\rho=|\Psi|^{2}$ and $\mathbf{v}_{\mathbf{q}}=(\hbar / 2 m) \nabla \log \rho$ is the "quantum" or osmotic velocity. The system describing the Madelung fluid in quantum mechanics is

$$
m\left(\mathbf{v}_{t}+(\mathbf{v} \cdot \nabla) \mathbf{v}\right)=-\nabla\left(U(x)+\frac{\hbar^{2}}{2 m} \frac{\nabla^{2} \sqrt{\rho}}{\sqrt{\rho}}\right), \quad \rho_{t}+\nabla(\rho \mathbf{v})=0,
$$

where $\mathbf{v} \equiv \mathbf{v}_{\mathbf{q}}$. Using complex velocity (1), we can write this system as the complex Burgers equation

$$
i \mathbf{V}_{t}+(\mathbf{V} \cdot \nabla) \mathbf{V}=-\frac{\hbar}{2 m} \Delta \mathbf{V}+\frac{1}{m} \nabla U
$$

for rotation-free motion, $\operatorname{curl} \mathbf{V}=0$. This nonlinear equation is C-integrable in the sense of Calogero [9], and using (1) as a complex version of the Cole-Hopf transformation [10], [11], we reduce it to the linear Schrödinger equation

$$
i \hbar \Psi_{t}=-\frac{\hbar^{2}}{2 m} \Delta \Psi+U \Psi
$$

1.2. Generalized Schrödinger equation. The dispersive part of the energy density for this equation is a quadratic form in the velocities:

$$
\epsilon_{0}=\frac{\hbar^{2}}{2 m} \nabla \bar{\Psi} \nabla \Psi=\frac{m \rho}{2} \overline{\mathbf{V}} \mathbf{V}=\frac{m}{2} \rho\left(\mathbf{v}_{c}^{2}+\mathbf{v}_{\mathrm{q}}^{2}\right) .
$$

To construct most the general extension of the dispersion, we can follow the postulate of analytic mechanics [12], where the kinetic term

$$
T=\frac{1}{2} \sum_{i, j} a_{i j}(q) \dot{q}_{i} \dot{q}_{j}
$$

is determined by a symmetric positive-definite quadratic form $a_{i j}$ with the generalized velocities $\dot{q}_{i}$, playing role of the Riemannian metric. We then generalize the dispersion

$$
\epsilon_{0}=\frac{m}{2} \rho\left(E \mathbf{v}_{c}^{2}+2 F \mathbf{v}_{\mathrm{c}} \mathbf{v}_{\mathrm{q}}+G \mathbf{v}_{\mathrm{q}}^{2}\right)
$$

in terms of the two-dimensional pseudo-Riemannian metric or first fundamental form $(E, F, G)$. This dispersion rewritten in terms of the wave function gives the nonlinear dispersive terms

$$
\epsilon_{0}=\frac{\hbar^{2}}{8 m}\left[2 E \nabla \bar{\Psi} \nabla \Psi+G \nabla|\Psi| \nabla|\Psi|-(E+2 i F)(\nabla \Psi)^{2} \frac{\bar{\Psi}}{\Psi}-(E-2 i F)(\nabla \bar{\Psi})^{2} \frac{\Psi}{\bar{\Psi}}\right] .
$$

Here, in addition to the standard linear dispersion, the second term represents the quantum potential contribution in form of the Fisher measure [13]. Auberson and Sabatier studied the linear Schrödinger equation with such a term [14]. The NLS equation with such a term was derived for the Jackiw-Teitelboim gravity in [6], [15], [16] and in plasma physics in [17]. The last two terms were introduced for a Hamiltonian generalization of the NLS equation by Malomed and Stenflo [18] and were studied for the resonance behavior in [19].

If we diagonalize dispersion term (8), then we obtain the standard linear dispersion

$$
H_{0}=\int d^{3} x\left(\frac{\rho \mathbf{V}_{c}^{2}}{2}+\frac{\rho \mathbf{V}_{\mathbf{q}}^{2}}{2}\right)=\frac{\hbar^{2}}{2 m} \int d^{3} x \nabla \bar{\psi} \nabla \psi
$$

for the elliptic case $g=E G-F^{2}>0$ and the nonlinear dispersion

$$
K_{0}=\int d^{3} x\left(\frac{\rho \mathbf{V}_{c}^{2}}{2}-\frac{\rho \mathbf{V}_{\mathbf{q}}^{2}}{2}\right)=\frac{\hbar^{2}}{2 m} \int d^{3} x(\nabla \bar{\psi} \nabla \psi-2 \nabla|\psi| \nabla|\psi|)
$$

for the hyperbolic case $g=E G-F^{2}<0$, where $\psi=\sqrt{\rho} e^{i S / \hbar}$ and

$$
\frac{\rho \mathbf{V}_{\mathbf{q}}^{2}}{2}=\nabla \sqrt{\rho} \nabla \sqrt{\rho}=\frac{1}{4} \frac{(\nabla \rho)^{2}}{\rho} .
$$


1.3. Scaling transformation as hyperbolic rotation of dispersion. Here, we consider dilatation of space coordinates applied to the above dispersions. Because of the normalization condition, the spatial coordinate scaling transformation $\mathbf{x} \rightarrow \mathbf{x}^{\prime}=e^{-\alpha / 2} \mathbf{x}$ applied to the probability density $\rho(x)$ implies [20]

$$
\rho \rightarrow \rho^{\prime}\left(x^{\prime}\right)=e^{\beta / 2} \rho\left(e^{\alpha / 2} x^{\prime}\right)
$$

where $\beta=\alpha$ for spatial dimensions $d=1$ and $\beta=3 \alpha$ for $d=3$. The invariance of the Hamilton-Jacobi equations implies

$$
S(x) \rightarrow S^{\prime}\left(x^{\prime}\right)=e^{-\alpha} S\left(e^{\alpha / 2} x^{\prime}\right)
$$

Because of these relations, the velocities transform as

$$
\mathbf{V}_{\mathrm{c}}(x)=e^{\alpha / 2} \mathbf{V}_{\mathrm{c}}^{\prime}\left(x^{\prime}\right), \quad \mathbf{V}_{\mathrm{q}}(x)=e^{-\alpha / 2} \mathbf{V}_{\mathrm{q}}^{\prime}\left(x^{\prime}\right)
$$

As a result, we obtain the hyperbolic rotation

$$
\begin{aligned}
& H_{0}=H_{0}^{\prime} \cosh \alpha+K_{0}^{\prime} \sinh \alpha, \\
& K_{0}=K_{0}^{\prime} \cosh \alpha+H_{0}^{\prime} \sinh \alpha,
\end{aligned}
$$

involving both elliptic and hyperbolic dispersions (10) and (11). Adding the nonlinear term to these dispersions, we introduce the two Hamiltonians

$$
H=\int d^{3} x\left[\frac{\hbar^{2}}{2 m}|\nabla \psi|^{2}-\frac{\Lambda}{8}|\psi|^{4}\right]
$$

and

$$
K=\int d^{3} x\left[\frac{\hbar^{2}}{2 m}|\nabla \psi|^{2}-\frac{\hbar^{2}}{m}|\nabla| \psi||^{2}-\frac{\Lambda}{8}|\psi|^{4}\right] .
$$

They generate evolutions in the two times $t$ and $\tau$,

$$
i \hbar \psi_{t}=\frac{\delta H}{\delta \bar{\psi}}, \quad i \hbar \psi_{\tau}=\frac{\delta K}{\delta \bar{\psi}}
$$

according to the corresponding NLS equation

$$
i \hbar \psi_{t}+\frac{\hbar^{2}}{2 m} \nabla^{2} \psi+\frac{\Lambda}{4}|\psi|^{2} \psi=0
$$

and the RNLS equation

$$
i \hbar \psi_{\tau}+\frac{\hbar^{2}}{2 m} \nabla^{2} \psi+\frac{\Lambda}{4}|\psi|^{2} \psi=2 \frac{\hbar^{2}}{2 m} \frac{\nabla^{2}|\psi|}{|\psi|} \psi
$$

The scaling transformation then acts on these dual Hamiltonians as a Lorentz boost transformation:

$$
\begin{aligned}
& H(\Lambda)=H^{\prime}\left(\Lambda^{\prime}\right) \cosh \alpha+K^{\prime}\left(\Lambda^{\prime}\right) \sinh \alpha, \\
& K(\Lambda)=K^{\prime}\left(\Lambda^{\prime}\right) \cosh \alpha+H^{\prime}\left(\Lambda^{\prime}\right) \sinh \alpha,
\end{aligned}
$$

where $\Lambda^{\prime}=\Lambda e^{-3 \alpha / 2}$ for $d=1$ and $\Lambda^{\prime}=\Lambda e^{-5 \alpha / 2}$ for $d=3$. 
1.4. RNLS. In one spatial dimension, Eq. (21) is just the cubic NLS equation, integrable for both signs of the coupling constant $\Lambda$ [21]. Dual equation (22) is the RNLS equation ( $\hbar=1, m=1 / 2)$,

$$
i \psi_{\tau}+\psi_{x x}+\frac{\Lambda}{4}|\psi|^{2} \psi=s \frac{|\psi|_{x x}}{|\psi|} \psi
$$

for $s=2[6]$. For this value of $s>1$, it reduces not to the usual NLS equation but to a reaction-diffusion (RD) system [15]. In this case, the model exhibits the resonance soliton phenomenon [6], [16]. For $s>1$, substituting $\psi=e^{R-i S}$, passing to $e^{+}=e^{R+S}$ and $-e^{-}=e^{R-S}$, and rescaling, we obtain the system

$$
\begin{aligned}
& -\frac{\partial e^{+}}{\partial t}+\frac{\partial^{2} e^{+}}{\partial x^{2}}+\frac{\Lambda}{4} e^{+} e^{-} e^{+}=0, \\
& \frac{\partial e^{-}}{\partial t}+\frac{\partial^{2} e^{-}}{\partial x^{2}}+\frac{\Lambda}{4} e^{+} e^{-} e^{-}=0 .
\end{aligned}
$$

The Madelung form of this system $\left(\Lambda=-2, \rho=1+\beta^{2}\right)$,

$$
\begin{aligned}
& \frac{\partial \rho}{\partial t}+\frac{\partial}{\partial x}(\rho u)=0, \\
& \frac{\partial u}{\partial t}+u \frac{\partial u}{\partial x}+\frac{\partial \rho}{\partial x}+\beta^{2} \frac{\partial}{\partial x}\left[\frac{1}{\rho} \frac{\partial^{2} \rho}{\partial x^{2}}-\frac{1}{2}\left(\frac{1}{\rho} \frac{\partial \rho}{\partial x}\right)^{2}\right]=0
\end{aligned}
$$

describes the propagation of long magnetoacoustic waves in a cold plasma of density $\rho$ moving with the velocity $u$ across the magnetic field [17].

Below, we show that RNLS equation (25) admits one more, non-Madelung hydrodynamic representation in the form of the Broer-Kaup and the classical Boussinesq systems [22], [23], which describe water wave propagation in a long narrow channel.

\section{Coupled heat equation}

System (26), (27) implies the conservation law

$$
\rho_{t}=\left(\rho\left(\log e^{+}\right)_{x}-\rho\left(\log e^{-}\right)_{x}\right)_{x},
$$

where the density is $\rho=-e^{+} e^{-}$. Using the identity $\rho_{x}=\rho\left(\log e^{+}\right)_{x}+\rho\left(\log e^{-}\right)_{x}$, we can eliminate one of the velocities and obtain continuity equations for $\rho$ that include only one field $e^{+}$or $e^{-}$. Combining with (26) or (27), we then obtain the independent system for $e^{+}$and $\rho$,

$$
\begin{aligned}
& -e_{t}^{+}+e_{x x}^{+}-\frac{\Lambda}{4} \rho e^{+}=0, \\
& \rho_{t}+\rho_{x x}=\left(2 \rho\left(\log e^{+}\right)_{x}\right)_{x},
\end{aligned}
$$

and a similar system for $e^{-}$and $\rho$ with the time reversal $t \rightarrow-t$. The first system describes the heat equation coupled to the "potential" $\rho$ and the continuity equation, and the second is the system for the backward heat equation. These two systems are related by the transformation $t \rightarrow-t$ and $e^{ \pm} \rightarrow e^{\mp}$.

The system admits the reduction $\rho \equiv 0$ and therefore reduces to the heat equation. The simplest solution of the heat equation then implies the solution $e^{+}=e^{v^{2} t / 4-v x / 2}, \rho=0$ of system (31), (32). It should be compared with the two-parameter dissipaton solution

$$
\begin{aligned}
& e^{+}=\left(\frac{8}{-\Lambda}\right)^{1 / 2} \frac{k e^{k^{2} t}}{\cosh k\left(x-v t-x_{0}\right)} e^{v^{2} t / 4-v x / 2}, \\
& \rho=\frac{8}{-\Lambda} \frac{k^{2}}{\cosh ^{2} k\left(x-v t-x_{0}\right)} .
\end{aligned}
$$


Here, the density $\rho$ is a traveling-wave soliton, and we have the dissipative soliton with a time-dependent amplitude for $e^{+}$.

\section{Hamilton-Jacobi-Bellman-type representation}

Dividing (31) by $e^{+}$and introducing the nonlinear change of variables $A^{+}(x, t)=2 \log e^{+}(x, t)$, similar to the one first made by Schrödinger in 1926 [24], we obtain the equivalent system

$$
\begin{aligned}
& -A_{t}^{+}+\frac{1}{2}\left(A_{x}^{+}\right)^{2}+A_{x x}^{+}-\frac{\Lambda}{2} \rho=0, \\
& \rho_{t}+\rho_{x x}=\left(\rho A_{x}^{+}\right)_{x}
\end{aligned}
$$

from system (31), (32). Equation (35) is well known in the theory of optimal stochastic control for continuous Markov processes and is called the Hamilton-Jacobi-Bellman (HJB) equation in this context [25]. It is a dynamic programming equation whose solution is the minimum value of some action functional. In our case, we have HJB equation (35) coupled to continuity equation (36). The system (35), (36) can be written as the Euler-Lagrange equation with the variational functional $S=\iint L d x d t$ and the Lagrangian density

$$
L=\frac{1}{2} \rho\left(A_{t}^{+}-A_{x x}^{+}\right)-\frac{1}{4} \rho\left(A_{x}^{+}\right)^{2}+\frac{\Lambda}{8} \rho^{2} .
$$

For one-dissipaton solution (33), we have system solution

$$
A^{+}=\left(k^{2}+\frac{1}{2} v^{2}\right) t-v x-2 \log \left[\cosh k\left(x-v t-x_{0}\right)\right]+\log \left(\frac{8 \sqrt{k}}{-\Lambda}\right)
$$

and $\rho$ given by (34).

\section{Broer-Kaup hydrodynamic representation}

The heat equation is naturally related to the Burgers equation by the Cole-Hopf transformation. This implies introducing the velocity field $v^{+}=\left(\log e^{+}\right)_{x}$, i.e., the drift velocity, and system (31), (32) then becomes

$$
\begin{aligned}
& v_{t}^{+}=\left(v_{x}^{+}+\left(v^{+}\right)^{2}-\frac{\Lambda}{4} \rho\right)_{x}, \\
& \rho_{t}+\rho_{x x}=\left(2 \rho v^{+}\right)_{x} .
\end{aligned}
$$

This system is known as the Broer-Kaup system [22], [23]. For $v^{-}=\left(\log e^{-}\right)_{x}$, it implies the anti-BroerKaup system with the time reversal $t \rightarrow-t$. For $\rho=0$, the systems reduce to the corresponding Burgers and anti-Burgers equations.

For $\rho \neq 0$, from dissipaton solution (33), we obtain the shock soliton

$$
v^{+}=-\frac{v}{2}-k \tanh k\left(x-v t-x_{0}\right)
$$

and soliton (34) of system (39), (40). 


\section{Classical Boussinesq representation}

Equation (39) can be represented in the Euler form. If we introduce the "pressure" function $p^{+}=$ $-\Lambda \rho / 4+v_{x}^{+}$, then we obtain the classical Boussinesq system

$$
\begin{aligned}
& -v_{t}^{+}+2 v^{+} v_{x}^{+}=-p_{x}^{+} \\
& p_{t}^{+}=v_{x x x}^{+}+\left(2 p^{+} v^{+}\right)_{x}
\end{aligned}
$$

from (39), (40). Similarly, introducing $p^{-}=-\Lambda \rho / 4+v_{x}^{-}$, we obtain the reverse-time classical antiBoussinesq system.

\section{Bilinear form and solitons}

Representing the two real functions $e^{+}$and $e^{-}$in terms of three real functions $g^{+}, g^{-}$, and $f, e^{ \pm}=$ $g^{ \pm} \sqrt{8 /(-\Lambda)} / f$, we consider the bilinear system of equations

$$
\begin{aligned}
& \left( \pm D_{t}-D_{x}^{2}\right)\left(g^{ \pm} \cdot f\right)=0, \\
& D_{x}^{2}(f \cdot f)=-2 g^{+} g^{-} .
\end{aligned}
$$

Any solution of system (44), (45) with

$$
\rho=\left(\frac{8}{-\Lambda}\right) \frac{g^{+} g^{-}}{f^{2}}=\left(\frac{8}{-\Lambda}\right)(\log f)_{x x}
$$

solves system (31), (32).

The solution of Broer-Kaup system (39), (40) is

$$
v^{+}=\frac{D_{x}\left(g^{+} \cdot f\right)}{g^{+} f}=\left(\log \frac{g^{+}}{f}\right)_{x}
$$

The solution of classical Boussinesq system (42), (43) is

$$
p^{+}=\left(\log \left(g^{+} f\right)\right)_{x}, \quad v^{+}=\left(\log \frac{g^{+}}{f}\right)_{x}
$$

6.1. One- and two-soliton solutions. The one-soliton solution of (44), (45) is given by $g^{ \pm}= \pm e^{\eta_{1}^{ \pm}}$, $f=1+e^{\eta_{1}^{+}+\eta_{1}^{-}+\phi_{1,1}}$, and $e^{\phi_{1,1}}=\left(k_{1}^{+}+k_{1}^{-}\right)^{-2}$, where $\eta_{1}^{ \pm} \equiv k_{1}^{ \pm} x \pm\left(k_{1}^{ \pm}\right)^{2} t+\eta_{1}^{ \pm(0)}$ and $k_{1}^{ \pm}$and $\eta_{1}^{ \pm(0)}$ are constants. It gives a kink-soliton solution for $v^{+}$in system (39), (40)

$$
v^{+}=\frac{k_{1}^{+}-k_{1}^{-}}{2}-\frac{k_{1}^{+}+k_{1}^{-}}{2} \tanh \frac{\eta_{1}^{+}+\eta_{1}^{-}+\phi_{11}}{2}
$$

and a soliton shape for the density $\rho$ (see Figs. 1 and 2)

$$
\rho=\frac{2}{-\Lambda} \frac{\left(k_{1}^{+}+k_{1}^{-}\right)^{2}}{\cosh ^{2}\left(\left(\eta_{1}^{+}+\eta_{1}^{-}+\phi_{11}\right) / 2\right)} .
$$

Introducing the parameters $k \equiv\left(k_{1}^{+}+k_{1}^{-}\right) / 2$ and $v \equiv-\left(k_{1}^{+}-k_{1}^{-}\right)$, we obtain solution (41). 


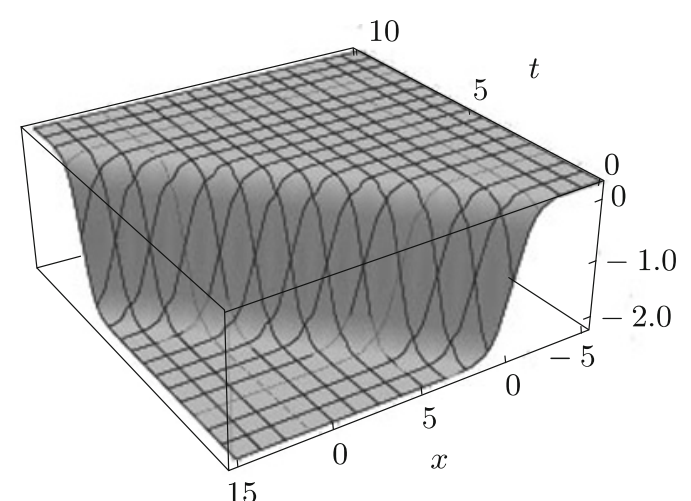

Fig. 1. Domain wall shape for the velocity field $v^{+}$.

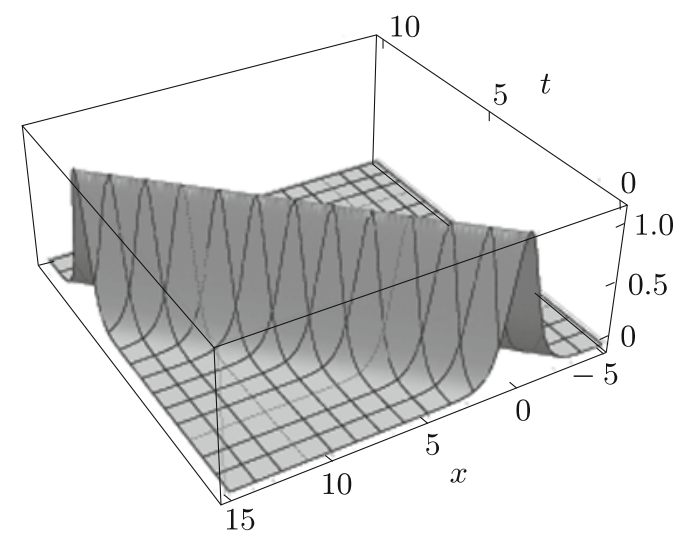

Fig. 2. Soliton shape for the density field $\rho$.

For classical Boussinesq system (42), (43), we have the kink-soliton solution

$$
p^{+}=\left(2 k-\frac{v}{2}\right)+k \tanh k\left(x-v t-x_{0}\right) .
$$

For the two-soliton solution, we have

$$
\begin{aligned}
g^{ \pm}= & \pm\left[e^{\eta_{1}^{ \pm}}+e^{\eta_{2}^{ \pm}}+\left(\frac{\breve{k}_{12}^{ \pm \pm}}{k_{21}^{ \pm \mp} k_{11}^{+-}}\right)^{2} e^{\eta_{1}^{+}+\eta_{1}^{-}+\eta_{2}^{ \pm}}+\left(\frac{\breve{k}_{12}^{ \pm \pm}}{k_{12}^{ \pm \mp} k_{22}^{+-}}\right)^{2} e^{\eta_{2}^{+}+\eta_{2}^{-}+\eta_{1}^{ \pm}}\right] \\
f=1 & +\frac{e^{\eta_{1}^{+}+\eta_{1}^{-}}}{\left(k_{11}^{+-}\right)^{2}}+\frac{e^{\eta_{1}^{+}+\eta_{2}^{-}}}{\left(k_{12}^{+-}\right)^{2}}+\frac{e^{\eta_{2}^{+}+\eta_{1}^{-}}}{\left(k_{21}^{+-}\right)^{2}}+\frac{e^{\eta_{2}^{+}+\eta_{2}^{-}}}{\left(k_{22}^{+-}\right)^{2}}+ \\
& +\left(\frac{\breve{k}_{12}^{++} \breve{k}_{12}^{--}}{k_{12}^{+-} k_{21}^{+-} k_{11}^{+-} k_{22}^{+-}}\right)^{2} e^{\eta_{1}^{+}+\eta_{1}^{-}+\eta_{2}^{+}+\eta_{2}^{-}}
\end{aligned}
$$

where $k_{i j}^{a b} \equiv k_{i}^{a}+k_{j}^{b}, \breve{k}_{i j}^{a b} \equiv k_{i}^{a}-k_{j}^{b}$, and $\eta_{i}^{ \pm} \equiv k_{i}^{ \pm} x \pm\left(k_{i}^{ \pm}\right)^{2} t+\eta^{ \pm(0)}$. This solution shows the resonance character of the soliton interaction (see Figs. 3 and 4 ).

6.2. Burgers reduction. The velocity $v^{+}$for the Broer-Kaup system in the Hirota form is $v^{+}=$ $g_{x}^{+} / g^{+}-f_{x} / f($ see $(47))$. If $g^{+}=$const, then $v^{+}=-(\log f)_{x}$, which implies the constraint $v_{x}^{+}=(\Lambda / 8) \rho$. Under this reduction, the Broer-Kaup system reduces to the Burgers equation

$$
v_{t}^{+}-2 v^{+} v_{x}^{+}+v_{x x}^{+}=0
$$




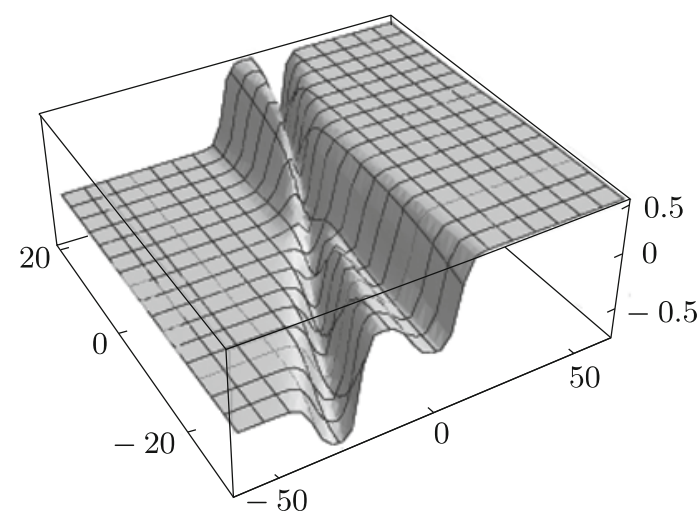

Fig. 3. The two-soliton velocity field $v^{+}$for $k_{1}^{+}=0.5, k_{1}^{-}=0.9, k_{2}^{+}=0.1$, and $k_{2}^{-}=0.35$.

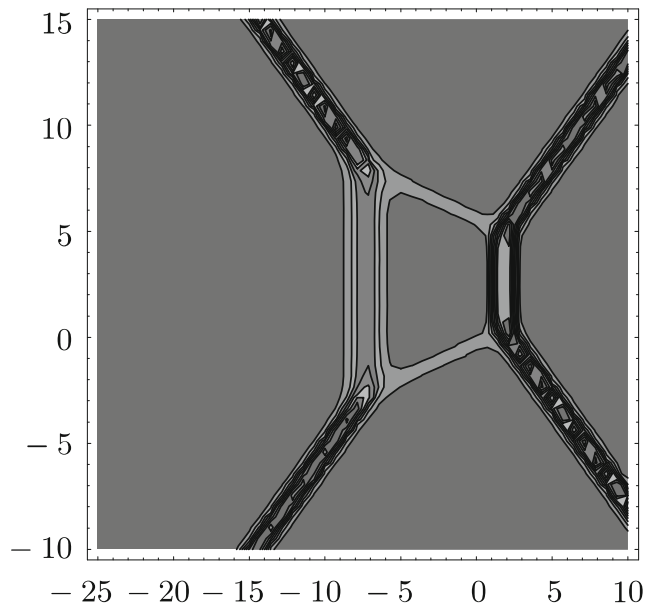

Fig. 4. Four-soliton resonance for the density field $\rho$ for $k_{1}^{+}=2, k_{1}^{-}=1, k_{2}^{+}=1, k_{2}^{-}=2$, and $d=16$.

Bilinear equation (44) then reduces to the heat equation

$$
f_{t}-f_{x x}=0
$$

and the above form $v^{+}=-(\log f)_{x}$ is just the Cole-Hopf transformation. On the level of the one-soliton solution with $k_{1}^{+}=0$, this gives just the Burgers shock-soliton solution. In the two-soliton case, setting $k_{1}^{+}=0$ and $k_{2}^{+}=0$, we then obtain two shock solitons

$$
v^{+}=-\frac{\left(2 / k_{1}^{-}\right) e^{\eta_{1}^{-}}+\left(2 / k_{2}^{-}\right) e^{\eta_{2}^{-}}}{1+\left(2 /\left(k_{1}^{-}\right)^{2}\right) e^{\eta_{1}^{-}}+\left(2 /\left(k_{2}^{-}\right)^{2}\right) e^{\eta_{2}^{-}}} .
$$

This solution describes the fusion of two shock solitons.

\section{Integrals of motion}

Here, we find integrals of motion for Broer-Kaup system (39), (40) with the vanishing boundary conditions $\rho \rightarrow 0$ as $|x| \rightarrow \infty$. The first integral of motion is the mass, and it follows just from (40):

$$
M=\int_{-\infty}^{\infty} \rho d x .
$$


The momentum conservation law

$$
\left(\rho v^{+}\right)_{t}=\left(\rho v_{x}^{+}-\rho_{x} v^{+}+2 \rho\left(v^{+}\right)^{2}-\frac{\Lambda}{8} \rho^{2}\right)_{x}
$$

implies the momentum integral

$$
P^{+}=-2 \int_{-\infty}^{\infty} \rho v^{+} d x
$$

The energy conservation law

$$
\left(\rho\left(v^{+}\right)^{2}-\rho_{x} v^{+}-\frac{\Lambda}{8} \rho^{2}\right)_{t}=\left(-3 \rho_{x}\left(v^{+}\right)^{2}+2 \rho\left(v^{+}\right)^{3}+\rho_{x x} v^{+}-\rho_{x} v_{x}^{+}-\frac{\Lambda}{2} \rho^{2} v^{+}+\frac{\Lambda}{4} \rho \rho_{x}\right)_{x}
$$

yields the energy integral

$$
E^{+}=2 \int_{-\infty}^{\infty}\left(\rho\left(v^{+}\right)^{2}+\rho v_{x}^{+}-\frac{\Lambda}{8} \rho^{2}\right) d x
$$

After Hirota's substitution, we have

$$
M=\int_{-\infty}^{\infty} \rho d x=\frac{8}{-\Lambda} \int_{-\infty}^{\infty}(\log f)_{x x} d x=\left.\frac{8}{-\Lambda}(\log f)_{x}\right|_{-\infty} ^{\infty}
$$

For the one-soliton solution, we have the mass

$$
M=\frac{8}{-\Lambda}\left|k_{1}^{+}+k_{1}^{-}\right|
$$

the momentum

$$
P^{+}=\frac{8}{-\Lambda}\left|k_{1}^{+}+k_{1}^{-}\right|\left(k_{1}^{-}-k_{1}^{+}\right)=M v
$$

where $v=k_{1}^{-}-k_{1}^{+}$, and the energy

$$
E=\frac{M v^{2}}{2}+\frac{\Lambda^{2}}{384} M^{3}
$$

The resonance conditions $M=M_{1}+M_{2}, P=P_{1}+P_{2}$, and $E=E_{1}+E_{2}$ computed with these integrals yield the velocity constraint

$$
\left|v_{1}-v_{2}\right|=-\frac{\Lambda}{8}\left(M_{1}+M_{2}\right)
$$

which reduces two-soliton solution (53) to the one-soliton solution.

\section{Geometric representation}

We introduce a pseudo-Riemannian metric in terms of the drift velocities [6]

$$
g_{00}=\rho v^{+} v^{-}, \quad g_{11}=-\rho, \quad g_{01}=\frac{1}{2} \rho\left(v^{-}-v^{+}\right) .
$$

For the RD system, this metric describes a pseudo-Riemannian surface of constant scalar curvature $R=\Lambda$. At a zero drift velocity, $v^{+}(x, t)=0$ or $v^{-}(x, t)=0$, this metric develops a horizon singularity and the black-hole-type picture [26]. This metric can be rewritten in terms of the Broer-Kaup hydrodynamics. For (39) and (40), we have

$$
g_{00}=v^{+}\left(\rho_{x}-\rho v^{+}\right), \quad g_{11}=-\rho, \quad g_{01}=\frac{1}{2} \rho_{x}-\rho v^{+} .
$$

At the horizon, $g_{00}=0 \rightarrow v^{+}=0$ or $\rho_{x}=\rho v^{+}$, and for the one-soliton solution, we obtain

$$
\tanh k\left(x-v t-x_{0}\right)=\mp \frac{v}{2 k},
$$


which admits two horizons for $|v|<2|k|$. The horizon condition $v^{+}=\phi_{x}=0$, implies an extremum of the velocity potential $\phi$.

\section{The third RD hierarchy flow and a higher hydrodynamic system}

The RD system is the second flow of the $S L(2, R)$ Ablowitz-Kaup-Newell-Segur hierarchy [27]. For the third flow of the hierarchy, we have

$$
\begin{aligned}
& e_{t}^{+}=e_{x x x}^{+}+\frac{3 \Lambda}{4} e^{+} e^{-} e_{x}^{+}, \\
& e_{t}^{-}=e_{x x x}^{-}+\frac{3 \Lambda}{4} e^{+} e^{-} e_{x}^{-} .
\end{aligned}
$$

Following a procedure similar to that for the $\mathrm{RD}$ system in terms of the hydrodynamic variables $v^{+}=$ $\left(\log e^{+}\right)_{x}$ and $\rho=e^{+} e^{-}$, we obtain the new system

$$
\begin{aligned}
& v_{t}^{+}=\left(v_{x x}^{+}+3 v^{+} v_{x}^{+}+\left(v^{+}\right)^{3}+\frac{3 \Lambda}{4} \rho v^{+}\right)_{x}, \\
& \rho_{t}=\left(\rho_{x x}-3 \rho_{x} v^{+}+3 \rho\left(v^{+}\right)^{2}+\frac{3 \Lambda}{8} \rho^{2}\right)_{x} .
\end{aligned}
$$

In the particular case $v^{+}=0$, it reduces to the Korteweg-de Vries equation. In another particular case $\rho=0$, this system reduces to a modification of the modified Korteweg-de Vries equation, which is exactly the third Burgers flow:

$$
v_{t}^{+}=v_{x x x}^{+}+3\left(v^{+}\right)^{2} v_{x}^{+}+\left(3 v^{+} v_{x}^{+}\right)_{x} .
$$

Substituting $e^{ \pm}=g^{ \pm} \sqrt{8 /(-\Lambda)} / f$, we obtain

$$
\begin{aligned}
& \left(D_{t}+D_{x}^{3}\right)\left(g^{ \pm} \cdot f\right)=0 \\
& D_{x}^{2}(F \cdot F)=-2 g^{+} g^{-}
\end{aligned}
$$

The solution of the system is then given by

$$
v^{+}=\frac{g_{x}^{+}}{g^{+}}-\frac{f_{x}}{f}, \quad \rho=\frac{8}{\Lambda} \frac{\partial^{2}}{\partial x^{2}} \log F
$$

For the one-soliton solution, we have the kink

$$
v^{+}=\sqrt{\frac{4 v^{2}-k^{2}}{12}}-\frac{k}{2} \tanh \frac{k}{2}\left(x-v t-x_{0}\right)
$$

and the soliton

$$
\rho=\frac{8}{4 \Lambda} \frac{k^{2}}{\cosh ^{2}(k / 2)\left(x-v t-x_{0}\right)},
$$


where $k=k_{1}^{+}+k_{1}^{-}$and $v=\left(k_{1}^{+}\right)^{2}-k_{1}^{+} k_{1}^{-}+\left(k_{1}^{-}\right)^{2}$. The last relation implies a restriction on the soliton speed, $|v|>|k| / 4$.

\section{The Broer-Kaup quantum fluid}

In this section, we show that the NLS equation can be represented as a new Broer-Kaup hydrodynamic system with a quantum potential. This representation is an alternative to the known Madelung representation of the NLS equation. If in the NLS equation

$$
i \psi_{t}+\psi_{x x}+\frac{\Lambda}{4}|\psi|^{2} \psi=0,
$$

we use the Madelung substitution $\psi=e^{R-i S}$ to introduce the density $\rho=|\psi|^{2}$, the center-of-mass or current velocity $V=-2 S_{x}$, and the quantum or osmotic velocity $V_{\mathrm{Q}}=2 R_{x}$, then we can obtain the Madelung fluid form of the NLS equation in terms of $\rho$ and $V$. By contrast, we here introduce the drift velocities $v^{+}=\left(V_{\mathrm{Q}}-V\right) / 2$ and $v^{-}=\left(V_{\mathrm{Q}}+V\right) / 2$. We can then rewrite the NLS equation in terms of $v^{+}$and $\rho$ as

$$
\begin{aligned}
& v_{t}^{+}=\left(v_{x}^{+}+\left(v^{+}\right)^{2}-\frac{\Lambda}{4} \rho-2 \frac{(\sqrt{\rho})_{x x}}{\sqrt{\rho}}\right)_{x}, \\
& \rho_{t}+\rho_{x x}=\left(2 \rho v^{+}\right)_{x}
\end{aligned}
$$

and the similar but time-reversed system in terms of $v^{-}$and $\rho$. These new equations can be regarded as a quantized Broer-Kaup systems.

\section{Modified Broer-Kaup system}

Here, we consider the next deformation of the MNLS-1,2 equations $(\epsilon= \pm 1)[28]$

$$
i \psi_{t}+\psi_{x x}+2 \gamma^{2}|\psi|^{2} \psi-i \mu_{0}(\epsilon+1)\left(\bar{\psi} \psi^{2}\right)_{x}+i \mu_{0}(\epsilon-1)(\bar{\psi} \psi) \psi_{x}=2 \frac{|\psi|_{x x}}{|\psi|} \psi
$$

It reduces to the RNLS equation at $\mu_{0}=0$ and to the resonance derivative NLS equations for $\gamma=0$ : the MNLS-1 equation with $\epsilon=1$ and the MNLS-2 equation with $\epsilon=-1$. Decomposing the complex function $\psi=e^{R-i S}$ and introducing the pair of real functions $e^{ \pm}=e^{R \pm S}$, we obtain the modified RD-1,2 systems $(\epsilon= \pm 1)$

$$
\mp e_{t}^{ \pm}+e_{x x}^{ \pm}-2 \gamma^{2} e^{+} e^{-} e^{ \pm} \pm \mu_{0}(\epsilon+1)\left(e^{+} e^{-} e^{ \pm}\right)_{x} \mp \mu_{0}(\epsilon-1)\left(e^{+} e^{-}\right) e_{x}^{ \pm}=0 .
$$

Further, introducing the density $\rho=e^{+} e^{-}$and velocity $v^{+}=\left(\log e^{+}\right)_{x}$, we obtain a new modified BroerKaup system

$$
\begin{aligned}
& v_{t}^{+}=\left(v_{x}^{+}+\left(v^{+}\right)^{2}-2 \gamma \rho+\mu_{0}(\epsilon+1) \rho_{x}+2 \mu_{0} \rho v^{+}\right)_{x} \\
& \rho_{t}+\rho_{x x}=\left(2 \rho v^{+}+\mu_{0}(\epsilon+2) \rho^{2}\right)_{x} .
\end{aligned}
$$

If $\mu_{0}=0$, then this system reduces to Broer-Kaup system (39), and if $\gamma=0$, then it reduces to another pair of modified Broer-Kaup systems depending on $\epsilon$.

Changing the functions $e^{ \pm}$to $E^{ \pm}$according the formulas

$$
e^{ \pm}=E^{ \pm} \exp \left(\mp \mu_{0}(\epsilon+1) \int_{-\infty}^{x} E^{+} E^{-} d x^{\prime}\right)
$$


and representing $E^{ \pm}=g^{ \pm} / f^{ \pm}$in terms of four real functions $g^{ \pm}$and $f^{ \pm}$, we obtain the bilinear representation of the modified RD-1,2 systems:

$$
\begin{aligned}
& \left(D_{t} \mp D_{x}^{2}\right)\left(g^{ \pm} \cdot f^{ \pm}\right)=0, \\
& D_{x}^{2}\left(f^{+} \cdot f^{-}\right)=\left(\mu_{0} D_{x}-2 \gamma^{2}\right)\left(g^{+} \cdot g^{-}\right), \\
& D_{x}\left(f^{+} \cdot f^{-}\right)=-\mu_{0} \epsilon g^{+} g^{-} .
\end{aligned}
$$

The one-soliton solution of the MNLS equation in a quantum potential or, equivalently, the one-dissipaton solution of the modified RD equation is given by the solution of Eqs. (87) $g^{ \pm}=e^{\eta^{ \pm}}, f^{ \pm}=1+e^{\phi^{ \pm}} e^{\eta^{+}+\eta^{-}}$, where $\eta^{ \pm}=k^{ \pm} x \pm\left(k^{ \pm}\right)^{2} t+\eta_{0}^{ \pm}$and

$$
e^{\phi^{ \pm}}=\frac{\mp \mu_{0}\left[k^{ \pm}(\epsilon-1)+k^{\mp}(\epsilon+1)\right]-2 \gamma^{2}}{2\left(k^{+}+k^{-}\right)^{2}} .
$$

For this solution to be regular, we restrict the parameters $k^{+}>\gamma^{2} / \mu_{0}$ and $k^{-}<-\gamma^{2} / \mu_{0}$. The velocity of our soliton $v=k^{-}-k^{+}$is then in the region $v<-\left(|k|+2 \gamma^{2} / \mu_{0}\right)$. For the velocity field, we obtain the shock soliton

$$
v^{+}=-\frac{v}{2}-k \tanh k\left(x-v t-x_{0}\right) .
$$

The corresponding mass density is given by

$$
\rho=\frac{k^{2} / \mu_{0}}{\sqrt{\left(v+2 \gamma^{2} / \mu_{0}\right)^{2}-k^{2}} \cosh k\left(x-v t-x_{0}\right)-\left(v+2 \gamma^{2} / \mu_{0}\right)} .
$$

Acknowledgments. This work was supported by TUBITAK (Project TBAG No. 110T679) and the Izmir Institute of Technology.

\section{REFERENCES}

1. E. Madelung, Naturwiss., 14, 1004-1004 (1926); Zeitschr. f. Phys., 40, 322-326 (1926).

2. D. Bohm, Phys. Rev., 85, 166-179, 180-193 (1952).

3. R. P. Feynman, The Feynman Lectures on Physics, Vol. 3, Quantum Mechanics, Addison-Wesley (1965).

4. S. Jin, C. D. Levermore, and D. W. McLaughlin, Commun. Pure Appl. Math., 52, 613-654 (1999).

5. J.-H. Lee, C.-K. Lin, and O. K. Pashaev, Chaos, Solitons Fractals, 19, 109-128 (2004).

6. O. K. Pashaev and J.-H. Lee, Modern Phys. Lett. A, 17, 1601-1619 (2002); arXiv:hep-th/9810139v2 (1998).

7. J. Matsukidaira, J. Satsuma, and W. Strampp, Phys. Lett. A, 147, 467-471 (1990).

8. L. D. Landau and L. M. Lifshitz, Course of Theoretical Physics [in Russian], Vol. 3, Quantum Mechanics: Non-relativistic Theory, Nauka, Moscow (1989); English transl. prev. ed., Pergamon, Oxford (1981).

9. F. Calogero, "C-integrable nonlinear partial differential equations," in: Important Developments in Soliton

Theory (A. S. Fokas and V. E. Zakharov, eds.), Springer, Berlin (1993), pp. 33-37.

10. J. D. Cole, Q. Appl. Math., 9, 225-236 (1951).

11. E. Hopf, Commun. Pure Appl. Math., 3, 201-230 (1950).

12. L. D. Landau and L. M. Lifshitz, Course of Theoretical Physics [in Russian] (3rd ed.), Vol. 1, Mechanics, Nauka, Moscow (1973); English transl., Pergamon, Oxford (1976).

13. R. R. Parwani and O. K. Pashaev, J. Phys. A, 41, 235207 (2008).

14. G. Auberson and P. C. Sabatier, J. Math. Phys., 35, 4028-4040 (1994).

15. O. K. Pashaev, Nucl. Phys. B Proc. Suppl., 57, 338-341 (1997).

16. O. K. Pashaev and J.-H. Lee, ANZIAM J., 44, 73-81 (2002).

17. J.-H. Lee, O. K. Pashaev, C. Rogers, and W. K. Schieff, J. Plasma Phys., 73, 257-272 (2007). 
18. B. A. Malomed and L. Stenflo, J. Phys. A, 24, L1149-L1153 (1991).

19. O. K. Pashaev, J.-H. Lee, and C. Rogers, J. Phys. A, 41, 452001 (2008).

20. L. Brenig, J. Phys. A, 40, 4567-4584 (2007); arXiv:gr-qc/0610142v2 (2006).

21. V. E. Zakharov and A. B. Shabad, Sov. Phys. JETP, 34, 62-69 (1972).

22. L. J. F. Broer, Appl. Sci. Res., 31, 377-395 (1975).

23. D. J. Kaup, Progr. Theoret. Phys., 54, 396-408 (1975).

24. E. Schrödinger, Annal. Phys., 384, 361-376 (1926).

25. R. Bellman, Dynamic Programming, Princeton Univ. Press, Princeton, N. J. (1957).

26. L. Martina, O. K. Pashaev, and G. Soliani, Class. Q. Grav., 14, 3179-3186 (1997); arXiv:hep-th/9710140v1 (1997); Phys. Rev. D, 58, 084025 (1998).

27. M. J. Ablowitz, D. J. Kaup, A. C. Newell, and H. Segur, Studies Appl. Math., 53, 249-315 (1974).

28. J.-H. Lee and O. K. Pashaev, "Bilinear representation for the modified nonlinear Schrödinger equations and their quantum potential deformations," in: Nonlinear Physics: Theory and Experiment II (Proc. of the Workshop, Gallipoli, Italy, 27 June-6 July 2002, M. J. Ablowitz, M. Boiti, F. Pempinelli, and B. Prinari, eds.), World Scientific, Singapore (2003), pp. 79-82. 